\title{
BREVE RELACION DE ALGUNĀS DE LAS PRINCIPALES PLANTAS Observadas en el Distrito Sur de la Baja California
}

\author{
Por el Prof. Maximino MARTINEZ
}

Como la descripción de la flora requeriría una extensión muy grande, aquí solamente se hace referencia a las plantas más útiles o más típicas de la región.

La Diamana (Turnera diffusa) es un arbusto de un metro de altura más o menos, de flores amarillas y hojas aromáticas, muy estimada tomada en forma de té como diurética y contra algunas enfermedades renales y vesicales. Se encuentra con mayor abundancia en la vertiente oriental del Distrito Sur, calculándose que se podrían cosechar anualmente 500 toneladas. Es objeto de exportación.

La Jojoba. (Simondsia californica) es un arbusto de 1 a $1.50 \mathrm{~m}$. de altura, de hojas opuestas y coriáceas, que se ve en el Distrito Sur, particularmente entre La Paz y San José del Cabo, por la vertiente oriental. Produce unos granos oscuros, de sabor parecido al de la avellana, aunque algo amargo. Contiene una substancia parecida al aceite, pero es en realidad una cera líquida, para la que se auguran muy importantes aplicaciones. En el Sur de los Estados Unidos a la fecha se están haciendo grandes plantaciones, en previsión de la gran demanda que habrá dentro de poco tiempo. Actualmente en la Baja California se explota en corta escala, usándose para preparar una especie de atole que llaman champurrado, muy agradable y nutritivo. Sin duda su cultivo sería de apreciable rendimiento.

El Palo Blanco (Lysiloma candida) es un arbolito de 8 a 10 metros, de corteza blanca. Se encuentra en el Distrito Sur, principalmente en las ca- 
ñadas. Las hojas son compuestas y las hojuelas son ovaladas u oblongas; el fruto mide 8 a $15 \mathrm{~cm}$. por 2.5 de ancho. La corteza es muy estimada como material curtiente y se hace gran consumo de ella en la Tenería Viosca. Oficialmente existen autorizadas 3,000 toneladas, comprendiendo por mitad a terrenos nacionales y a terrenos particulares. Como no se cultiva se está agotando rápidamente. Actualmente derriban el árbol o bien se arranca la corteza dejándolo en pie completamente inutilizado. Convendría cortar la corteza solamente de un lado en una tira longitudinal, para conservar la vida del árbol y esperar la posible regeneración de la parte cortada. Además, sería conveniente sembrarlo por semillas en su propio terreno. En vez de eso, que sería lo más indicado, están procuranıo aclimatar el dividivi, de lo que no hay necesidad, sobre todo cuando los industriales informan que el Palo blanco es superior.

\section{El Mauto. (Lysiloma divaricaia). Es}

un arbolillo de 6 a 8 metros, con

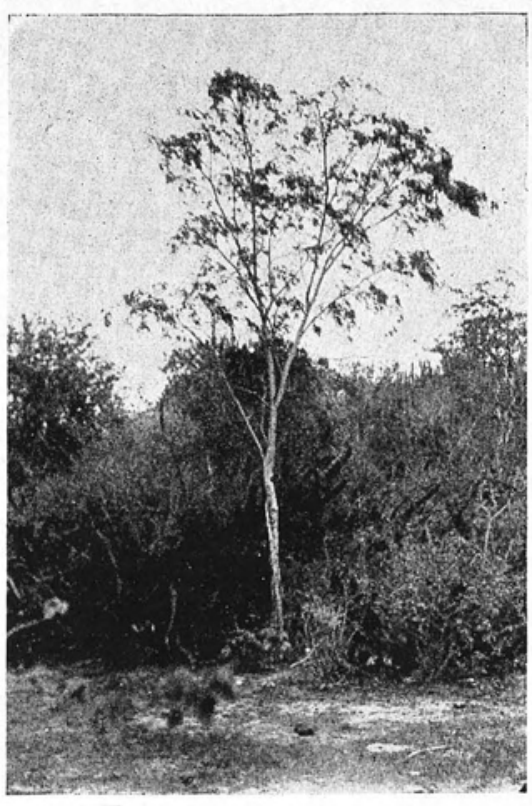

1 Palo Blanco (Lysiloma candida)

las hojas doblemente divididas en hojuelas de $3 \mathrm{~mm}$. El fruto es una vaina aplastada, de unos $10 \mathrm{~cm}$. Lo observé entre Santiago y El Triunfo, pero lo hay en otros lugares, principalmente en las estribaciones de la sierra. La corteza es muy estimada para curtir vaquetas y gamuzas.

El Orégano (Lippia Palmeri). Es planta aromática muy apreciada como condimento. Mide de uno a dos metros, con hojas de 15 a 20 mm., con las' florecillas blancas. Se observa en la región de Miraflores; cerca de Todos Santos, hay otra especie (Lippia formosa) y otra en Comondú y Mulegé (Lippia barbata). Es objeto de exportación.

Palo de arco. (Tecoma stans angustata). Es un arbusto de hojas compuestas y flores amarillas, muy vistosas. Las ramas se usan para hacer cacaxtles (huacales) y para sostener los muros de las casas rústicas. El cocimiento de las hojas se emplea contra la diabetes. Con corta diferencia es la planta conocida en el centro del país con el nombre de tronadora. Es común de La Paz hacia el Sur, y prospera mejor en los sitios algo húmedos.

Los Cardones. (Pachycereus Pringlei). Cactáceas centenarias que alcanzan de 10 a 15 metros con tronco hasta de un metro de diámetro. Se ramifican diversamente y dan unos frutos espinosos, comestibles. Se ven en todo el Distrito Sur formando a veces masas tupidas que cubren dilatadas ex- 


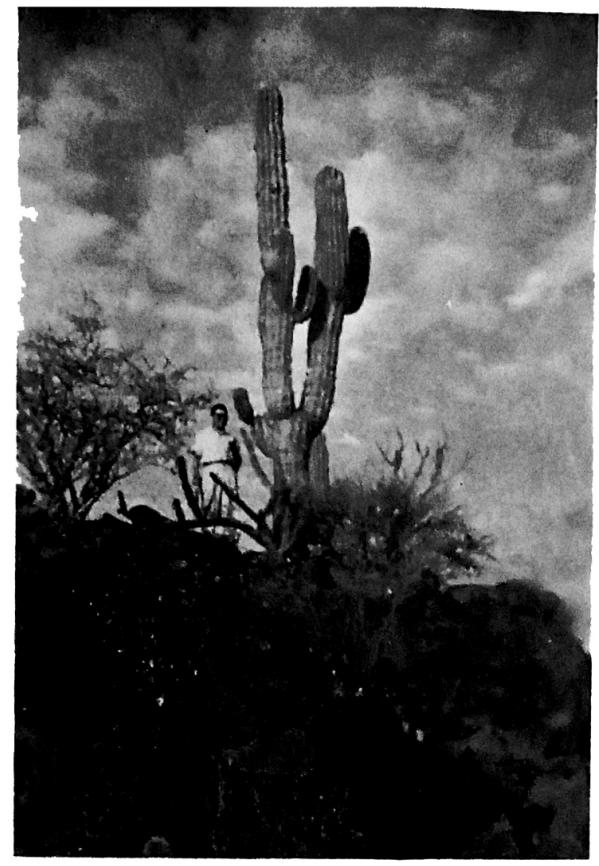

2 Cardón (Pachycereus Pringlei)

amarillo y con ellos hacen una especie de jalea. Se ve en todo el Distrito.

Garambullo (L o p ho c e r e u s Schotti). Alcanza unos 3.50 metros y sus tallos siempre erguidos; las espinas cercanas a la extremidad son largas y delgadas, en tanto que las inferiores son gruesas y cortas. Las flores se producen en el límite de esas dos clases de espinas. El fruto es rojo y comestible.

Ocotillo (Fouquieria splendens). Es planta hasta de 10 metros de altura, compuesta de numerosos tallos que parten desde la base, no ramificados y totalmente cubiertos de espinas. Las hojas aparecen solamente durante algunas épocas. Las flores son rojas. Se ve en todo el Distrito y es la misma especie que se encuentra en las zonas áridas del Norte del país. Una especie de (Fouquieria Burragei) observada a unos $8 \mathrm{~km}$. al norte de La Paz, tiene flores casi blancas. tensiones. El centro de los tallos es le ñoso y suele emplearse para construc ciones. Hay una especie, llamada Cau dón barbón (Pachycereus pecten-abc riginum) de menores dimensiones, qu se encuentra en la región Sur de la $\mathrm{P}_{\epsilon}$ nínsula

Pitaya agria (Manchaerocereus gur mosus). Es cactácea de tallos extend dos de 2 metros o más de largo y de 1 a $12 \mathrm{~cm}$. de diámetro armados de fue tes espinas. Su fruto es comestible Abunda en todo el Distrito y caracter: za a todas las regiones áridas. La part carnosa sirve para embarbascar.

Pitaya dulce (Lemaireocereus thus beri). Se distingue de la anterior pc sus tallos erguidos, que alcanzan $d$ 6 a 8 metros, con espinas meno peligrosas. Los frutos son de color rojo. 


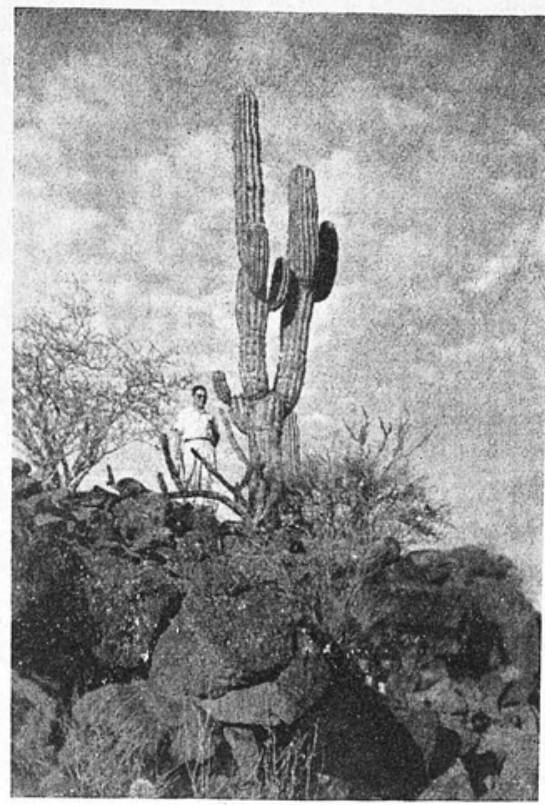

2 Cardón (Pachycereus Pringlei)

amarillo y con ellos hacen una especie de jalea. Se ve en todo el Distrito.

\section{Garambullo (L o phocere u s Scho-}

tti). Alcanza unos 3.50 metros y sus tallos siempre erguidos; las espinas cercanas a la extremidad son largas y delgadas, en tanto que las inferiores son gruesas y cortas. Las flores se producen en el límite de esas dos clases de espinas. El fruto es rojo y comestible.

Ocotillo (Fouquieria splendens). Es planta hasta de 10 metros de altura, compuesta de numerosos tallos que parten desde la base, no ramificados y totalmente cubiertos de espinas. Las hojas aparecen solamente durante algunas épocas. Las flores son rojas. Se ve en todo el Distrito y es la misma especie que se encuentra en las zonas áridas del Norte del país. Una especie de (Fouquieria Burragei) observada a unos $8 \mathrm{~km}$. al norie de La Paz, tiere flores casi blancas. tensiones. El centro de los tallos es le ñoso y suele emplearse para construc ciones. Hay una especie, llamada Cas dón barbón (Pachycereus pecten-abc riginum) de menores dimensiones, qu se encuentra en la región Sur de la $\mathrm{Pe}$ nínsula.

Pitaya agria (Manchaerocereus gum mosus). Es cactácea de tallos extend dos de 2 metros o más de largo y de 1 a $12 \mathrm{~cm}$. de diámetro armados de fue tes espinas. Su fruto es comestible Abunda en todo el Distrito y caracter za a todas las regiones áridas. La part carnosa sirve para embarbascar.

Pitaya dulce (Lemaireocereus thur beri). Se distingue de la anterior po sus tallos erguidos, que alcanzan $\mathrm{d}$ 6 a 8 metros, con espinas meno peligrosas. Los frutos son de color rojo

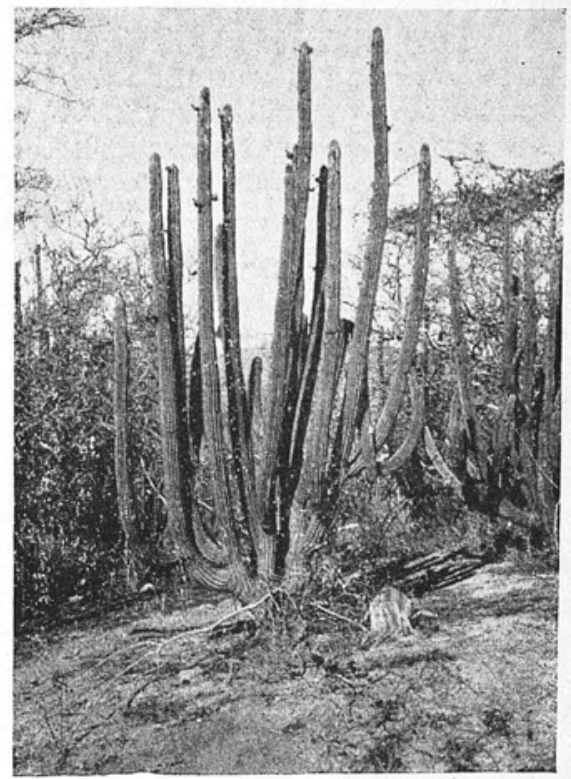

3 Pitaya dulce (Lemaireocereus Thurberi) 


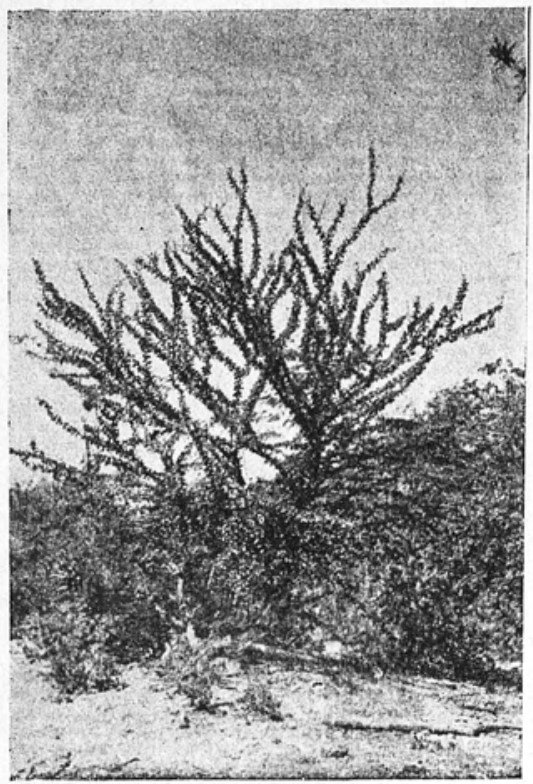

4 Palo Adán (Fouquieria peninsulare)

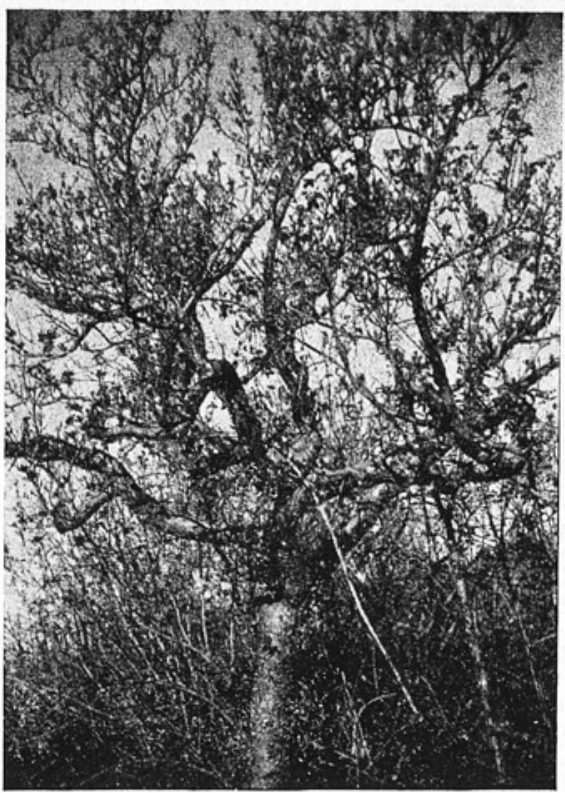

5 Torote (El phirium microphyllum)

Palo Adán (Fouquieria peninsularis). Es parecido al ocotillo, distinguiéndose en que tiene un tallo definido y con las ramas divididas y extendidas. Su corteza es amarillenta y sirve, macerada, para desmanchar ropa, especialmente de lana. Abunda en todo el Distrito.

Torote colorado (Bursera microphylla). Es arbolillo de 3 a 6 metros, de ramas irregulares y retorcidas, con la corteza rojiza, apergaminada. Hay otra especie con la corteza cenicienta y de ramas más tortuosas al que llaman torote blanco, cuya goma es venenosa. Hay aún otras especies de Bursera, llamadas unas Palo Colorado, Copal, etc., no bien definidas, por falta de material botánico, pues me tocó verlas sin flores ni frutos, y en algunos casos sin hojas.

Lomboy (Jatropha cinerea). Es una de los arbustos más comunes en todo el Distrito Sur. Alcanza unos 4 o 5 metros y en sus hojas son anchas y polimorfas, de tamaño variable y de superficie cenicienta. Sus flores son pequeñas y rojas. Al trozar las ramas sale un líquido transparente que los campesinos aplican sobre las heridas leves para su rápida cicatrización. Deja en la ropa mancha indeleble. Hay otra especie llamada Lomboy colorado (Jatropha vernicosa) que se distingue de la anterior por sus hojas angulosas y brillantes, su tallo oscuro y por sus flores blancas. Le dan iguales usos.

Chirinola (Machaerocereus eruca). Es el "cacto que camina". Los tallos, de uno a dos metros, se encuentran tendidos sobre la arena, donde introducen sus raíces. Las espinas son fuertes y peligrosas. Al producirse en 
la extremidad un nuevo retoño, mientras éste crece, la parte posterior se seca, dando la impresión de que el cacto ha caminado. Se observa al Sur de Comondú y por el rumbo de La Purísima hacia el Pacífico.

Entre las Cactáceas es notable el Ferocactus Wislizeni que alcanza dos metros de altura, con fuertes espinas, sobre todo la del centro, que es aplanada y ganchuda. Se encuentra en abundancia entre San Ignacio y Calmallí.

Datilillo o Dátil cimarrón. (Yucca valida). Se encuentra particularmente de La Paz hacia el Norte, en terrenos calizos y muy áridos. De la parte interior del tronco extraen una fibra que se usa para rellenar colchones; las hojas pueden utilizarse para extraer fibra de buena calidad. Se observó en cantidad explotable de Los Mártires y El Porvenir hacia San Andrés, en una extensión de cerca de 30 kilómetros, y también en una región situada de 30 a $50 \mathrm{~km}$. al N. O. de La Paz, en el camino a Santa Rosalía.

El Palo Verde. (Cercidium peninsulare). Se caracteriza por tener ese color en sus tallos y ramas. Estas son espinosas y los renuevos sirven de alimento al ganado, lo mismo que los írutos.

El Palo Brea. (CERCIDIUM TORREYANA). Es muy parecido al anterior; pero con la espina menos fuerte y la vaina aplastada. Ambos se ven en todo el Distrito.

El Dipúa (Cercidium sp). Se asemeja a los anteriores, pero es seguramente otra especie no bien determinada. Se encuentra solamente de La Paz hacia el Norte y es muy estimado como el mejor forraje, pues los campesinos aseguran que el ganado que lo come engorda rápidamente. No encontré flores ni frutos.

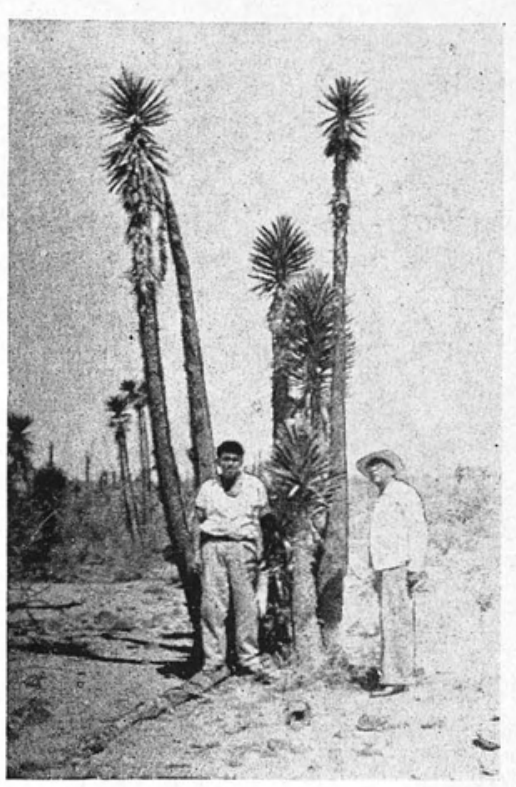

6 Datilillo (Yucea valida)

El Mezquite dulce. (Prosopis chilensis). (-P. julilora). Es el mismo que se conoce en el Centro del País. Vi, además, el mezquite amargo, llamado así por el sabor de sus frutos. Posiblemente es una variedad del anterior.

Palo fierro (Pithecollobium confine). Es arbusto espinoso de 1.50 a 3 metros. Sus frutos son vainas leñosas y oscuras, de superficie roñosa y miden unos $12 \mathrm{~cm}$. de largo. La madera es muy dura y las semillas se usan como café.

Las cuatro plantas siguientes son venenosas:

Candelilla. (Pedilanthus macrocarpus). Es planta común en todo el 
Distrito. Como las hojas caen pronto, se presenta como un conjunto de tallos cenicientos, de un metro o algo más de altura por unos $2.5 \mathrm{~cm}$. de diámetro. Las flores y los frutos tienen color rojo. Al herirse los tallos dejan escapar un líquido lechoso que, a pequeñas dosis es un purgante enérgico, y en cantidad mayor puede causar la muerte. No es la misma planta que en Coahuila y otros lugares del Norte se llama Candelilla y que produce cera.

Cacachila. (Karwinskia Humboldtiana). Es un arbolillo conocido en varios lugares de la República con el nombre de Tullidora o Capulincillo. Las semillas causan, tanto al hombre como a los animales, una parálisis difícil de curar. Existe en las estribaciones de la Sierra de La Laguna.

Caribe. (Jatropha angustidens). Mide más o menos un metro de altutura; sus hojas son anchas y palmadas, cubiertas, lo mismo que toda la planta de numerosos aguijones urticantes, que causan intensa molestia. Abunda de La Paz hacia el Sur, desde pocos metros sobre el nivel del mar hasta las estribaciones de la Sierra, a 1,000 metros o algo más.

Palo de la flecha. (Sebastiania pavoniana). Es un arbusto muy ramificado desde su base, llegando a unos 7. metros de altura. Si se le hiere, suelta un líquido lechoso, sumamente cáustico, que destruye la piel y ocasiona la ceguera si cae en los ojos. Los antiguos indios envenenaban sus flechas con ese jugo. A veces los pescadores echan ramas trozadas en el agua para cegar a los peces, pero es práctica peligrosa y prohibida. En las semillas suele encontrarse una larva que devora su contenido, quedándose encerrada en la cubierta mientras sufre su transformación; dicha larva, al contacto de la mano o al calor del sol se contrae bruscamente, haciendo saltar la semilla Esta peligrosa planta, que debería exterminarse, se ve entre La Paz y Todos Santos, ya cerca de esta población. También se ven algunos ejemplares en los suburbios de La Paz.

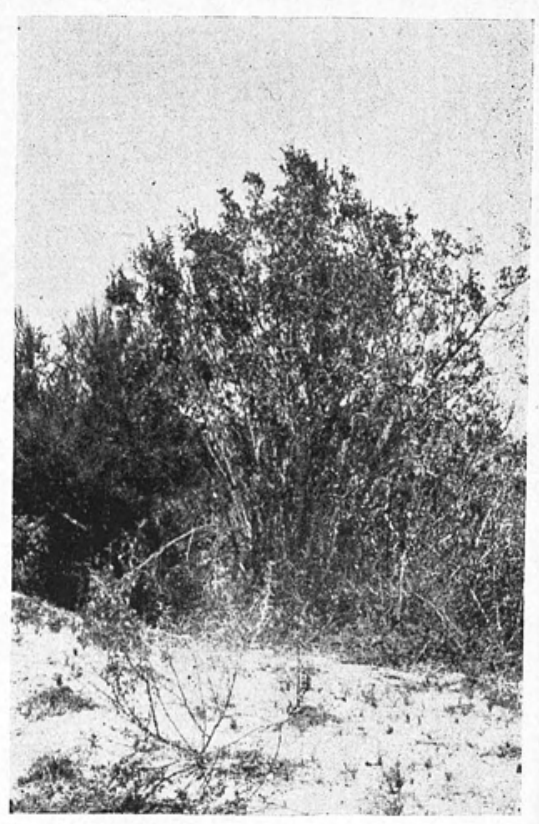

En la Serranía son plantas notables:

7 Palo de la Flecha (Sebastiania pavoniana)

El Zalate. (Ficus Palmeri). Pertenece al grupo de los Amates, tan co. munes en los climas cálidos del Centro y Sur del país. Sus raíces son muy grandes, de color blanquizco y se abrazan a las rocas, tomando formas fantásticas. Produce frutos pequeños, comestibles, semejantes a los higos. 
Cacalosúchil. (Plumiera acutifolia). Es arbusto silveste, de 4 a 8 metros de altura, con las hojas amontonadas en la extremidad de las ramillas, y las flores blancas y vistosas. El tallo suelta al herirse un jugo lechoso, que tiene alguna proporción de hule, pero no en cantidad explotable. Se observa en las estribaciones de la Sierra de La Laguna.

Güérivo. (Populus monticola). Es una especie de álamo, de 15 metros de altura. Su madera es rojiza y muy estimada para hacer yugos, muebles, etc. Se ve en las estribaciones de la Sierra de La Victoria, de preferencia en las barrancas.

Madroño. (Arbutus peninsularis). Se ve en los lugares elevados de ia Sierra, dintinguiéndose por su corteza rojiza, que se desprende en placas delgadas. La madera es quebradiza y poco durable.

Piñonero. (Pinus cembroides). Este pino, muy conocido en el país, desde los Estados fronterizos hasta Puebla, alcanza en el Valle de La Laguna, mayores dimensiones, de 10 a 18 metros. Es extraordinariamente abundante pero la semilla no se aprovecha por la dificultad para sacarla de esa región.

Encinos. Observé dos especies en las estribaciones de La Sierra y en el Valle de La Laguna (Quercus idonea y Q.devia).

Papache. (Randia Watsoni). Es un arbusto espinoso con las espinas dispuestas en cruz, por lo que en muchos lugares le dicen cruceto. Las hojas tiene propiedades astringentes.

Plantas diversas:

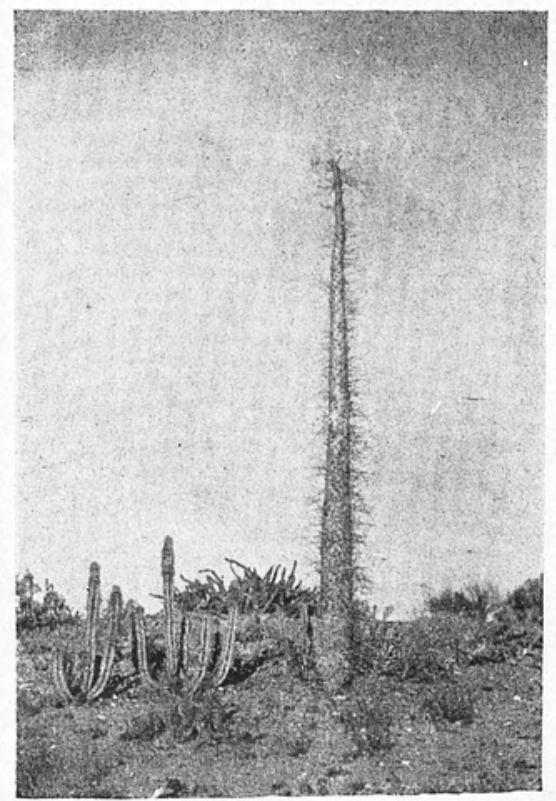

S Cirio (Idria columnaris)

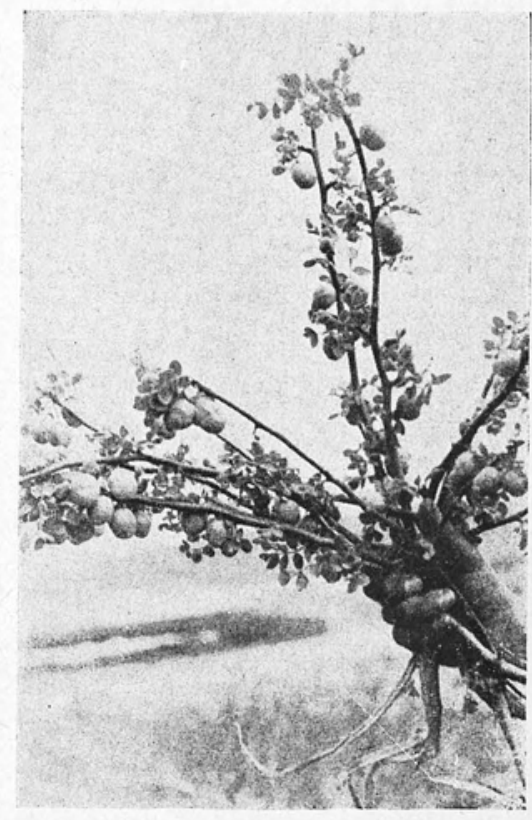

y Ciruelo (Cyrtocarpa edulis) 
Ciruelo. (Cyrtocarpa edulis). Es un arbolillo de 3 a 6 metros, con ramas irregulares; que produce una enorme cantidad de frutos comestibles, ovales, algo picudos, de color amarillento y de sabor agridulce, algo astringente. Abunda de La Paz hacia el Sur, pudiendo recogerse los frutos por toneladas. La semilla, llamada chunique, es también comestible, y tiene un sabor parecido al del piñón.

Cirios. (Idria columnaris). Plantas raras, de forma columnar, que se encuentran solamente en la Baja California, principalmente entre los paralelos 28 y 29. Los tallos alcanzan de 10 a 15 metros y son de color verde amarillento, revestidos de penachos de espinas, entre las que aparecen ocasionalmente las hojas. Las flores, casi blancas, se presentan en racimos colocados en la extremidad y semejan la flama de un cirio.

Romerillo. (Hymenoclea monogyra). Es planta de 1 a 15 metros de altura, ramosa, de hojas muy delgadas y aromáticas, que se ve en todos los arroyos. En épocas de sequía sirve de forraje.

Marinola. (Solanun Hindsianum). Es planta espinosa, de un metro a un metro y medio de altura, con flores violáceas. Se ve por todas partes, pero con mayor abundancia de La Paz hacia el Sur.

Incienso. (Encelia farinosa). Es planta de un metro aproximadamente, con hojas cenicientas, casi blancas y flores amarillas, muy común en todo el Distrito Sur. Produce una resina aromática.

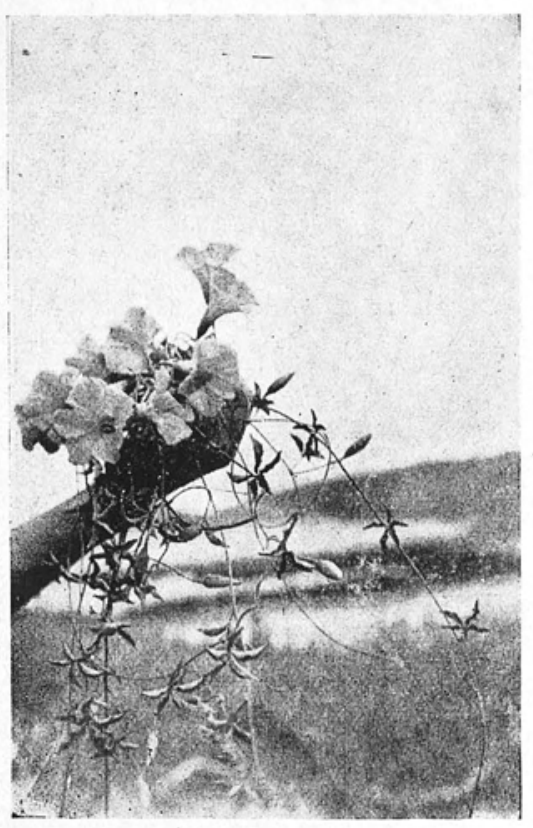

10 Yuca (Operculina aurea)

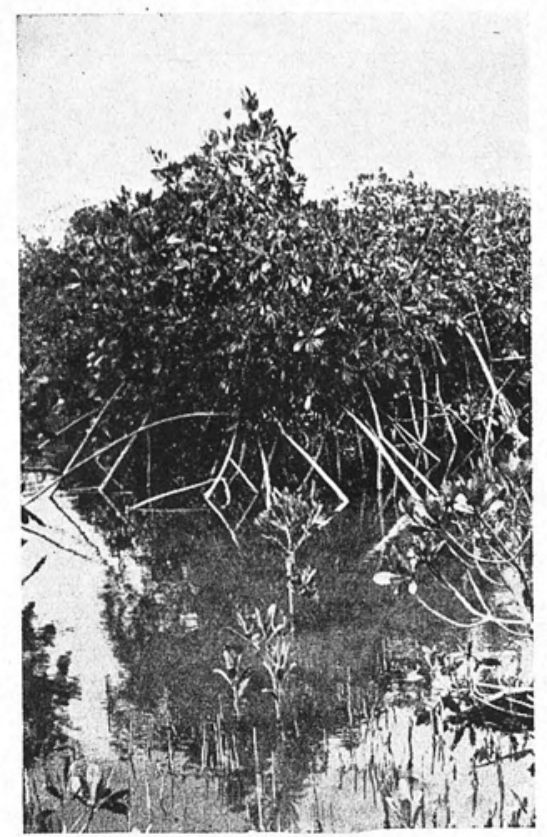

11 Mangle (Rhizophora mangle) 
Otatave. (Vallesia glabra). Es un arbusto de 2 a 8 metros, con ramas algo glaucas y hojas lanceoladas, flores blancas, muy pequeñas y frutos ovales, blancos y jugosos, de sabor dulzón. Los campesinos afirman que las gallinas que los comen "se vuelven más ponedoras".

Palo zorrillo. (Cassia occidentalis). Es un arbolillo de 4 a 6 metros con hojas de olor especial, desagradable; las flores tienen color amarillo y los frutos son vainas aplastadas. Es el mismo árbol que en varios lugares de la República se llama bricho o hendiondillo. Se observa de La Paz hacia el Sur, en las estribaciones de la Sierra. Proporciona madera para construcciones.

Orchilla. (Roccella tinctoria). Es un liquen que abunda en el litoral del Pacífico. Se encuentra revistiendo las ramas de los arbustos, principalmente los ocotillos. Antiguamente se usaba para fabricar tintes y era objeto de activo comercio, pero la introducción de las anilinas le quitó toda importancia.

Yuca. (Operculina aurea). Es una enredadera de hojas digitadas que cubre los arbustos con sus flores amarillas, muy vistosas. Solamente se observa de La Paz hacia el Sur.

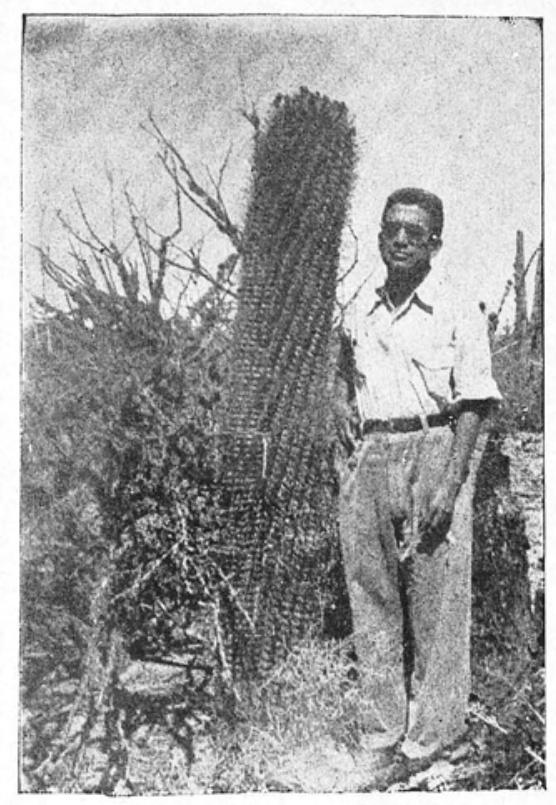

12 Bisnaga (Ferocactus Wislizeni)

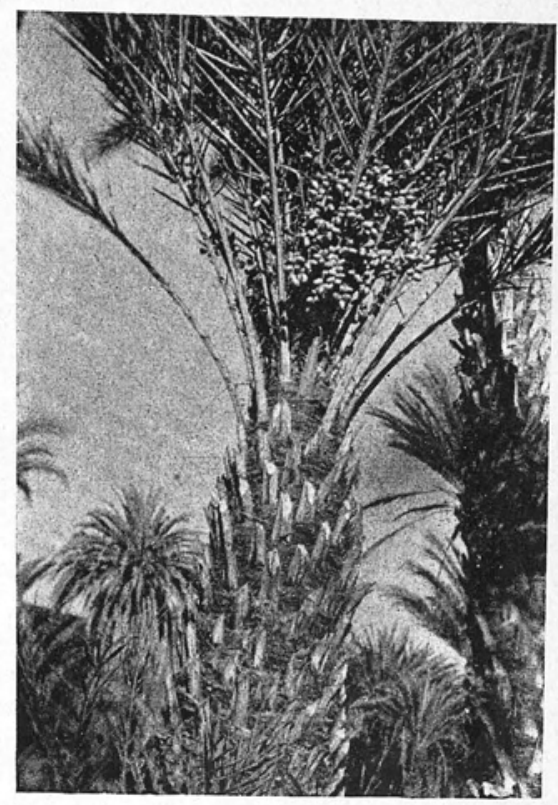

13 Datilera (Phoenix dactylifera)

San Miguel. (Antigonon leptopus). Es otra enredadera de flores color carmín o rosado, muy ornamentales. Como la anterior, se observa de La Paz hacia el Sur. 
Palmas de abanico. Se ven dos especies: Washingtonia filifera yWashingtonia sonorae. Usan las hojas para techar las casas, colocándolas a manera de tejas.

Manglares. En las costas se ven los manglares, habiendo el mangle rojo (Rhizophora mangle) el mangle salado (Avicennia nitida) y el mangle cenizo (Conocarpus erecta). El más importante es el primero porque su corteza es tanante.

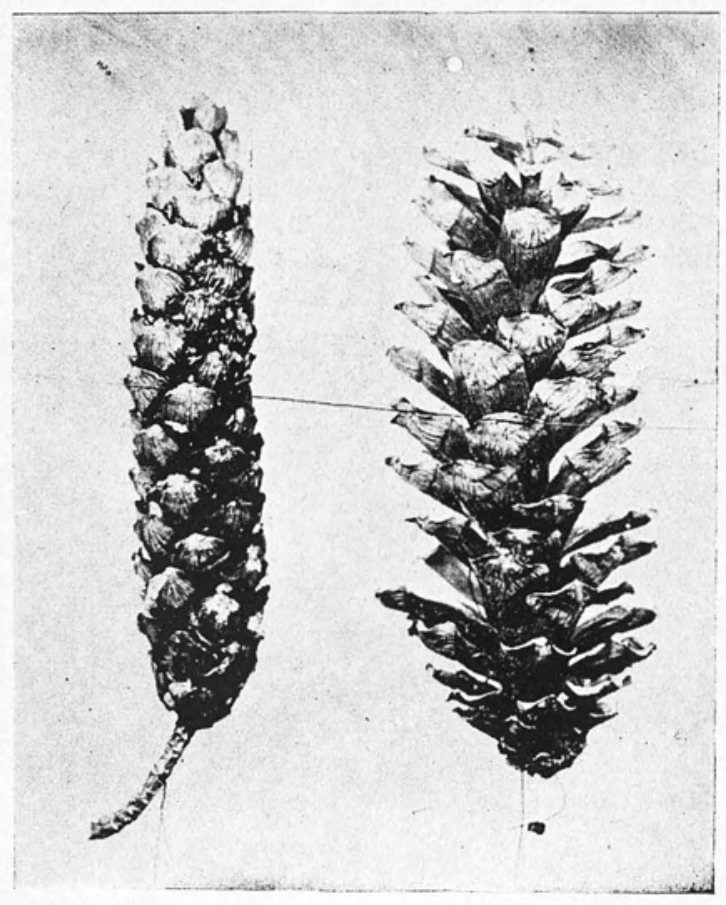

Pinus Strobus chiapensis, euya zona de sitribucıón se extiende de Veracruz a Oaxaca y Chiapas. 


\section{SINONIMIA VULGAR Y CIENTIFICA DE ALGUNAS PLANTAS DE BAJA CALIFORNIA}
Alcajer.
Pereskiopsis Porteri (T. S. Brand). Br. \&. Rose.
Ahuejote.
Salix lasiolepis Benth.
Algodón cimarrón.
Gossypium Davidsoni Kellog.
Algorón ciinarrór. Amargoso.
Amole.
Gossypium Harknessii T. S. Brand.
Castela peninsularis Rose.
Cactáceas.
Salicáceas.
Malváceas.
Malváceas.
Stegnosperma halimifolium Benth. Fitolacáceas.
Bebelama.
Bumelia occidentalis Hemsl.
Biche.
Biznaga.
Cassia biflora L. Ferocactus Wislizeni (Engelm) Br.
Bledo. $\&$ Rose.
Celosia floribunda A. Gray.
Brasil.
Haematoxylum brasiletto Karst.
Sapotáceas.
Leguminosas.
Cactáceas.
Amarantáceas.
Leguminosas.

Karwinskia Humboldtiana (Roem. 4 Shult). Zuc.

Plumiera acutifolia Poir.

Pedilanthus macrocarpus Benth. Euforbiáceas.

Pachycereus Pringlei (S. Wats). Br. ¿. Kose.

Cactáceas.

Pachycereus pecten-aboriginum Eng). Br. \&. Rose.

Cactáceas.

Caribe.

Celosa.

Cirio.

Ciruelo.

Ciruelo de monte.

Cochal.

Colorín.

Confituria.

Copal.
Jatropha angustidens. (Torr). Muell Euforbiáceas.

Mimosa Xanti A. Gray.

Idria columnaris Kellog.

Cyrtocarpa edulis T. S. Brand.

Cyrtocarpa edulis T. S. Brand.

Myrtillocactus cochal (Orcutt- Br. \&. Rose.

Erythrina flabelliformis Kearn.

Lantana camara L.

Elaphrium sp.
Leguminosas.

Fouquieriáceas.

Anacardiáceas.

Anacardiáceas.

Cactáceas.

Leguminosas.

Verbenáceas.

Burseráceas. 
Chamiso.

Chamiso.

Chicura.

Chilicote.

Chirinol.

Cholla.

Chuchupate.

Chuparrosa.

Damiana.

Dátil.

Dátil cimarrón.

Datilillo.

Dipúa.

Encino.

Encino negro.

Encino roble.

Escoba amarga.

Espuela del dicblo.

Estafiate.

Frutilla.

Gallinitas.

Garabato blanco.

Garambullo.

Gobernadora.

Gordolobo.

Guaco:

Guatamote.

Guayabillo.

Guayparín.

Giiérivo.

Giirote negro.

Huérivo.

Huizache.

Incienso.

Huizapol.

Jarilla.

Jarilla de loma.

Jícama.

Jito.

Jojoba.

Jumete.

Junco.
Atriplex canescens. (Pursh) Nutt.

Atriplex linearis S. W ats. y otros.

Franseria ambrosioides Cav.

Erythrina flabelliformis Kearn.

Machaerocereus eruca (T. S. Brand) Br. \&. Rose.

Opuntia cholla Engelm.

Quenopodiáceas.

Quenopodiáceas.

Compuestas.

Leguminosas.

Cactáceas.

Cactáceas.

Arracacia Brandegeei Colt. \& Rose. Umbelíferas.

Calliandra californica Benth.

Leguminosas.

Turnera diffusa. Willd

Turneráceas.

Phoenyx dactylifera L. (Cultivada). Palmas.

Yuca valida T. S. Brand.

Yuca valida T. S. Brand.

Cercidium sp.

Liliáceas.

Liliáceas.

Leguminosas.

Quercus Brandegeei Gold.

Quercus devia Gold.

Quercus idonea Gold.

Baccharis sarotroides A. Gray.

Martynia altaefolia Benth.

Artemisia sp.

Lycium Rechii A. Gray.

Fagáceas.

Fagáceas.

Fagáceas.

Compuestas.

Martiniáceas.

Compuestas.

Solanáceas.

Mascagnia macroptera (Moc. \&. Sess). Nied.

Celtis reticulata Torr.

Lophocereus Schottii (Engelm). Br \&. Rose.

Covillea tridentata (D. C.). Vail. Gnaphalium crenatum Greenm.

Cleomella longipes Torr.

Baccharis glutinosa Pers.

Dodonaea viscosa Jaca.

Maba latifolia St.

Populus monticola T. S. Lrand.

Arrabidaea sp.

Populus monticola T. S. Brand.

Acacia farnesiana (L). Willd.

Encelia farinosa A. Gray.

Cenchrus Palmeri Vasey.

Dodonaea viscosa Jacq.

Dodonaea viscosa Jacq.

Ipomoea jicama T. S. Brand.

Forchammeria Watsoni Rose.

Simondsia californica Nutt.

Asclepias subulata Decais.

Parkinsonia aculeata L.
Sapindáceas.

Ulmáceas.

Cactáceas.

Zigofiláceas.

Compuestas.

Caparidáceas.

Compuestas.

Sapindáceas.

Diospiráceas.

Salicáceas.

Bignoniáceas.

Salicáceas.

Leguminosas.

Compuestas.

Poáceas.

Sapindáceas.

Sapindáceas.

Convolvuláceas.

Caparidáceas.

Buxáceas.

Asclepiadáceas.

Leguminosas. 
Junco.

Parkinsonia microphylla Torr.

Junco marino.

Parkinsonia aculeata L.

Leguminosas.

Leguminosas.

Levántate don Juan Nicotiana glauca Graham.

Liga.

Euphorbia Xanti Engelm.

Jatropha cinerea (Ort). Muell.

Lomboy.

Jatropha cinerea (Ort). Muell.

Jatropha vernicosa T. S. Brand.

Lomboy brilloso.

Lomboy colorado.

Jatropha vernicosa T. S. Brand.

Madroño.

Malva rosa.

Mangle.

Mangie blanco.

Mangle cenizo.

Mangle colorado.

Mangle salado.

Manzanita.

Maravilla.

Mariola.

Matacora.

Arbutus peninsularis Rose \&. Gold.

Melochia tomentosa L.

Rhizophora mangle L.

Conocarpus erecta $\mathrm{L}$.

Conocarpus erecta $\mathrm{L}$.

Rhizophora magle L.

Avicennia nitida Jacq.

Solanáceas.

Euforbiáceas.

Euforbiáceas.

Euforbiáceas.

Euforbiáceas.

Euforbiáceas.

Malpighia diversifolia T. S. Brand.

Mirabilis Wrightiana Gray.

Solanum Hindsianum Benth.

Jatropha spathulata Muell. Arg.

Ericáceas.

Esterculiáceas.

Rizoforáceas.

Combretáceas.

Combretáceas

Rizoforáceas.

Verbenáceas.

Malpigiáceas.

Nictagináceas.

Solanáceas.

Euforbiáceas.

Mauto.

Melón coyote.

Mezquite amargo.

Mezquite dulce.

Nandimbo.

Lysiloma divaricata (Jacq.) Macbr.

Ibervillea sonorae (Sw) Greenm.

Prosopis juliflora velutina (Woot) Sarg.

Prosopis juliflora (Sw). D. C.

Ehretia tinifolia L. (cultivada).

Fouquieria splendens Engelm.

Ocotillo.

Leguminosas.

Cucurbitáceas.

Ocotillo de flor blan. ca. Fouquieria Burragei Rose.

Orchilla.

Roccella tinctoria Ach.

Leguminosas.

Leguminosas.

Borragináceas.

Fouquieriáceas.

Orégano (Región de Comondú). Lippia barbata T. S. Brand.

Orégano (Región de T. Santos).

Lippia formosa T. S. Brand.

Orégano (Región de Miraflores).

Ojo de pajarito.

Otatave.

Lippia Palmeri S. Wats.

Fouquieriáceas.

Líquenes.

Verbenáceas.

Verbenáceas.

Verbenáceas.

Dolicholus phaseoloides (Sw) Kuntze Leguminosas.

Vallesia glabra (Cav). Link.

Apocináceas.

Palma.

Erythea Brandegeei Purpus.

Palma de abanico.

Palma negra.

Palmilla.

Palo Adán.

Palo amarillo.

Palo de arco.

Washingtonia filifera Wendl.

Erythea Brandegeei Purpus.

Nolina Beldingi T. S. Brand.

Fouquieria peninsulare Nash.

Esenbeckia flava T. S. Brand

Tecoma stans angustata Rehder.

Fenicáceas.

Fenicáceas.

Fenicáceas.

Liliáceas.

Fouquieriáceas.

Rutáceas.

Bignoniáceas. 
Palo blanco.

Palo brea.

Palo chino.

Palo ebx.

Palo escopeta.

Palo de la flecha.

Palo fierro.

Palo San Juan.

Palo verde.

Palo zorrillo.

Papache.

Piñón.

Pitaya agria.

Pitaya dulce.

Raja matraca.

Roble.

Romerillo.

Salate.

Salvia.

Salvia.

San Miguel.

Sauz.

Talayote.

Tamariz.

Tarais.

Timbe.

Toji.

Torote bianco.

Torote colorado.

Tripa de aura.

Uña de gato.

Vainoro.

Vcrra prieta.

Vid.

Vinorama.

Yerba del venado.

Yedra.

Yuca.

Zalate.

Zapotillo.

Zarza mora.

Zaya.
Lysiloma candida T. S. Erand.

Leguminosas.

Cercidium Torreyana (S. Wats) Sw. Leguminosas.

Pithecollobium mexicanum Rose.

Leguminosas.

Leguminosas.

Albizzia occidentalis T. S. Brand. Leguminosas. Sebastiania pavoniana Muell.

Euforbiáceas.

Pithecollobium confine S. T. Brand. Leguminosas.

Forchammeria Watsoni Rose.

Cercidium peninsulare Rose.

Cassia occidentalis L.

Randia Watsoni Rob.

Pinus cembroides Zucc.

Machaerocereus gummosus (Engelm). Br. \&. Rose.

Lemaireocereus Thurberi (Engelm). Br. \&. Rose).

Caparidáceas.

Leguminosas.

Leguminosas.

Rubiáceas.

Pináceas.

Cactáceas.

Cactáceas.

Wilcoxia striata (T. S. Brand). Br. \&. Rose.

Quercus idonea. Goldm.

Cactáceas.

Fagáceas.

Hymenoclea monogyra Torr \&. Gray. Compuestas.

Ficus Palmeri S. Wats.

Hyptis laniflora Benth.

Salvia similis T. S. Brand.

Moráceas.

Labiadas.

Labiadas.

Antigonon leptopus Hook \&. Arn.

Salix Bonplandiana Kunth.

Rothockia cordifolia A. Gray.

Tamarix gallica L. (cultivado).

Salix taxifolia H. B. K.

Poligonáceas.

Salicáceas.

Asclepiadáceas.

'Tamaricáceas.

Salicácer $x$ s.

Acacia angustissima (Mill). Kuntze.

Phoradendron commutatum Trel. .

Elaphrium sp.

Elaphrium microphyllum (A Gray). Rose.

Ipomoea pes-caprae (L). Swartz.

Olneya tesota A. Gray.

Celtis reticulata Torr.

Cordia Greggii Torr.

Vitis vinifera L. (Cultivada).

Acacia farnesiana (L). Willd.

Porophyllum gracile Benth y otras sp.

Rhus radicans L.

Operculina aurea (Kellog) House.

Ficus Palmeri S. Wats.

Maba latifolia St.

Rubus oligospermus T. S. Brand. Amoreuxia sp.
Leguminoseds.

¿orantáceas.

Burseráceas.

Sìrseráceas.

Convolvulácecis.

Leguminosas.

Ulmáceas.

Borragináceas.

Vitáceas.

Leguminosas.

Compuestas.

Anacardiáceas.

Convolvuláceas.

Moráceas

Diospiróreas.

Rosáceas.

Coclospermáceas. 